\title{
Erratum to: Parasitism and the demography of wheat stem sawfly larvae, Cephus cinctus
}

\author{
Robert K. D. Peterson - Micaela Buteler • \\ David K. Weaver · Tulio B. Macedo - Zhitan Sun ·
}

Oscar Gerardo Perez • Godshen R. Pallipparambil

Published online: 10 May 2011

(C) International Organization for Biological Control (IOBC) 2011

\section{Erratum to: BioControl \\ DOI 10.1007/s10526-011-9357-7}

The original publication contains the following errors:

Results section, first sentence of last paragraph: "(100\% irreplaceable mortality)" should read "(100 - percent irreplaceable mortality)"

Acknowledgements section, first name "M. Hoflund" should read "M. Hofland"

The online version of the original article can be found under doi:10.1007/s10526-011-9357-7.

R. K. D. Peterson $(\bowtie) \cdot$ M. Buteler · D. K. Weaver .

T. B. Macedo · Z. Sun · O. G. Perez ·

G. R. Pallipparambil

Montana State University, Bozeman, USA

e-mail: bpeterson@montana.edu 